\title{
Microdosimetric characterisation of radiation fields for modelling tissue response in radiotherapy
}

\author{
He Wang ${ }^{1}$, Oleg N Vassiliev ${ }^{2}$ \\ ${ }^{I}$ Department of Radiation Physics, The University of Texas MD Anderson Cancer Center, Houston, Texas, USA. \\ ${ }^{2}$ Department of Medical Physics, Mary Bird Perkins Cancer Center, Baton Rouge, Louisiana, USA.
}

Received December 31, 2013; Revised February 07, 2014; Accepted February 19, 2014; Published Online February 23, 2014

\section{Original Article}

\begin{abstract}
Purpose: Our overall goal is the development of an approach to model tissue response to radiotherapy in which a tissue is viewed as a statistical ensemble of interacting cells. This involves characterisation of radiation fields on the spatial scale of subcellular structures. On this scale, the spatial distribution of radiation energy imparted to tissue is highly non-uniform and should be characterised in statistical terms. Microdosimetry provides a formalism developed for that purpose. This study addresses limitations of the standard microdosimetric approach to modelling tissue response by introducing two new characteristics that include additional information in a form convenient for this application. Methods: The standard microdosimetric approach is based on the concept of a sensitive volume (SV) representing a target volume in the cell. It is considered in isolation from other SVs, implying that energy depositions in different SVs are statistically independent and that individual cells respond to radiation independent of each other. In this study, we examined the latter approximation through analysis of correlation functions. All calculations were performed with Geant4-DNA Monte Carlo code. Results: We found that for some realistic scenarios, spatial correlations of deposited energy can be significant. Two new characteristics of radiation fields are proposed. The first is the specific energy-volume histogram $(\mathrm{zVH})$, which is a microscopic analogue of the dose-volume histogram. The second describes the probability distribution of deposited energies in two SVs without assuming statistical independence between the SVs. Numerical examples for protons and carbon ions of therapeutic energies are presented and discussed. Conclusion: We extended the microdosimetric approach to modelling tissue response by including additional important characteristics and presented them in a more conventional radiotherapy format.
\end{abstract}

Keywords: Tissue response; Microdosimetry; Hadron therapy

\section{Introduction}

Microdosimetry plays an important role in radiation therapy research. The microdosimetric approach to modelling the response of biological systems to radiation has been particularly useful in hadron therapy. Several studies have applied it to calculating the relative biological effectiveness (RBE) of therapeutic proton and carbon beams. ${ }^{1-8}$ Furthermore, microdosimetric techniques are indispensable for evaluating dose equivalent outside the treatment field, including neutrons. ${ }^{9-11}$ The microdosimetric approach is also applicable to

Corresponding author: Oleg N Vassiliev, PhD; 4950 Essen Lane, Baton Rouge, LA, 70809, USA. Email: ovassiliev@marybird.com

Cite this article as:

Wang H, Vassiliev ON. Microdosimetric characterisation of radiation fields for modelling tissue response in radiotherapy. Int J

Cancer Ther Oncol 2014; 2(1):020116. DOI: 10.14319/ijcto.0201.16 modelling the biological effects of sparsely ionizing radiations. It has been applied to RBE analysis of radiation sources used in brachytherapy ${ }^{12-14}$ as well as external beam therapy with photon and electron beams. ${ }^{15,} 16$ Microdosimetric concepts are also a foundation of several models of cell survival, for example. ${ }^{17-22}$

Nevertheless, microdosimetry has had a rather limited impact on clinical practice. It is not mentioned in a recent task group report from the American Association of Physicists in Medicine on biologically related models for treatment planning. ${ }^{23}$ Part of the reason for this omission is the lack of a fully established methodology for applying microdosimetry to radiotherapy. For example, the question of whether single-event microdosimetric spectra $f_{1}(y)$ (where $y$ is the lineal energy) or multiple-event spectra $f(y \mid D)$ should be used at the relatively high dose levels typical for radiotherapy has been sub- 
ject to debate. ${ }^{24,} 25$ Another unsettled matter is the size of the sensitive volume (SV). Usually a size on the order of $1 \mu \mathrm{m}$ is used, but this number has been constantly challenged. A recent study ${ }^{22}$ strongly suggested that for modelling cell survival, the SV size should be much smaller, about $10 \mathrm{~nm}$.

There is another problem that, to the best of our knowledge, has not yet been addressed. The standard assumption in microdosimetry is that the response of a biological system, such as the number of surviving cells, can be deduced from a microdosimetric spectrum for a single SV. This implies that energy deposition in a given SV is statistically independent from energy depositions in other SVs, either within the same cell or in neighbouring cells, and that individual cells respond to radiation independent of one another. In the present study, we report data showing strong correlations between energy depositions in two SVs that do not attenuate with increasing distance between the SVs, up to $10 \mu \mathrm{m}$ (the maximum distance that we tested).

As for the cellular response, it is well known that cells in tissues are not independent. In fact, radiation-induced bystander effects have received much attention in recent years. ${ }^{26}$ The bystander effect, however, is outside the scope of the present study, which is limited to the physics only. Furthermore, although the definition of a microdosimetric spectrum includes the probability of no energy deposition in an SV ${ }^{27}$, this probability is rarely reported or considered in radiobiological models. In hadron therapy, especially for carbon beams, it is significant even for the relatively large (on the cellular scale) volumes on the order of $1 \mu \mathrm{m}^{3}$. This probability characterises the spatial heterogeneity of energy deposition on the microscopic scale and, therefore, is an important parameter that should be considered, especially when modelling the tumour control probability (TCP). To address these problems, we introduce in this study new parameters for characterising statistical properties of microscopic patterns of energy deposition. These parameters are similar to the standard microdosimetric quantities ${ }^{27}$ but include additional information in a form convenient for modelling tissue response in radiotherapy.

\section{Methods and Materials}

This study is based on Monte Carlo simulations performed with Geant4-DNA, release 9.6 (May 17, 2013). The physics implemented in the code is well described elsewhere. ${ }^{28}$ The physics models needed for the present study are presently available only for one material, liquid water. This is one of the standard materials that is considered tissue equivalent for the purposes of radiobiological modelling. For modelling direct interactions of radiation with cellular targets, it would be preferable to use physics data, such as interaction cross sections, that are specific for each target type (e.g., a DNA molecule). Such data presently is not available owing largely to the complexity of the physics of such interactions, especially at the very low energies that are important for this study. The interaction processes included in the model, for all particles that we simulated, and the respective energy ranges in which the process is active, are as follows:

- $\quad$ Electrons: elastic scattering (7.4 eV-1 MeV), electronic excitation (9 eV-1 MeV), ionization (11 eV-1 $\mathrm{MeV})$, vibrational excitation $(2 \mathrm{eV}-100 \mathrm{eV})$, attachment $(4 \mathrm{eV}-13 \mathrm{eV})$

- Protons: electronic excitation (10 eV-100 MeV), ionization $(100 \mathrm{eV}-100 \mathrm{MeV})$, electron capture (100 $\mathrm{eV}-100 \mathrm{MeV}$ )

- Hydrogen atoms: electronic excitation (10 eV-500 $\mathrm{keV})$, ionization (100 eV-100 MeV), charge increase $(100 \mathrm{eV}-100 \mathrm{MeV})$

- Carbon ions: ionization $\left(0.5 \mathrm{MeV} / \mathrm{u}-10^{6} \mathrm{MeV} / \mathrm{u}\right)$; the model does not include electronic excitations or charge exchange processes (electron capture or loss), and therefore the results we present for carbon beams should be viewed qualitatively only.

Charge transfer processes, electron capture for protons, and charge increase for hydrogen atoms become important at very low energies, near the track end. They become the dominant inelastic processes at particle energies lower than approximately $100 \mathrm{keV} .{ }^{29}$ In electron capture processes, an electron from a water molecule is transferred to the incident proton to form a neutral hydrogen atom. Conversely, in the charge increase processes, the electron is stripped from the neutral hydrogen atom and, normally, is ejected in a forward direction with approximately the same velocity as the incident hydrogen atom. ${ }^{29}$ Charge transfer processes are no less important for low-energy carbon ions. In this case, however, the physics is much more complex, in part because a carbon ion can be in any of several charge states, from a fully stripped ion (charge +6$)$ to a neutral atom. These processes are not included in the current version of Geant4-DNA. We will add that the number of proton therapy centers significantly exceeds the number of active carbon therapy facilities. Therefore, results for proton beams are more important at the present time.

Electron histories were terminated when their kinetic energy fell below $11 \mathrm{eV}$ (the ionization threshold in the model). The residual range of an $11 \mathrm{eV}$ electron is on the order of $10 \mathrm{~nm}$ according to Francis et al. ${ }^{30}$ However, this number is associated with high uncertainty. Nikjoo and Lindborg ${ }^{31}$, for example, reported that the range of a $100 \mathrm{eV}$ electron is only 4.9 $\mathrm{nm}$. Terminating electron history at $11 \mathrm{eV}$ was unlikely to introduce significant errors in our study because the spatial resolution of the results we reported was $10 \mathrm{~nm}$. Histories of protons and carbon ions were terminated after they travelled 
a distance of about $10 \mu \mathrm{m}$. Therefore, no energy cutoffs were applied to these particles.

Energy depositions were tallied in a box sized $1 \mu \mathrm{m} \times 1 \mu \mathrm{m}$ in the $\mathrm{X}$ and $\mathrm{Y}$ directions and $10 \mu \mathrm{m}$ in the $\mathrm{Z}$ direction. The initial direction of particle travel was parallel to the $\mathrm{Z}$ axis. The size of the simulation volume exceeded that of the tally box by $20 \mathrm{~nm}$ in all directions. The $20 \mathrm{~nm}$ of padding was needed to achieve equilibrium of secondary electrons produced by protons and carbon ions throughout the tally box, which was estimated using Monte Carlo simulations. The material of the simulation volume was liquid water. The tally box was divided into $10 \mathrm{~nm}$ cubic voxels. Energy depositions were tallied separately for individual voxels.

A parallel beam of particles travelling in the positive $\mathrm{Z}$ direction was incident on one side of the simulation volume. The beam size was $1.04 \mu \mathrm{m} \times 1.04 \mu \mathrm{m}$, which coincides with the size of the simulation volume in the $\mathrm{XY}$ direction. The number of incident particles in one history was randomly sampled from a Poisson distribution. The average number of incident particles per history (parameter of the Poisson distribution) was chosen so that the average dose in the tally box was $2 \mathrm{~Gy}$ per history. This value (2 Gy) is representative of a typical dose delivered to the tumor volume in one treatment fraction. In hadron therapy, the dose is usually prescribed in Gy (RBE). In this study, however, we specified the absorbed dose in Gy only. Using Gy (RBE) would require specifying an RBE value, which, conceptually, would contradict our overall purpose of improving tissue response models.

The following quantities were calculated:

- Probability distribution $f(\varepsilon)$ of deposited energy $\varepsilon$ in individual $10 \mathrm{~nm}$ voxels. This included the probability of no energy deposition, $\varepsilon=0$. The energy resolution was $1 \mathrm{eV}$. Generally, $f(\varepsilon)$ depends on the dose and should be written as $f(\varepsilon \mid D)$. However, all results reported in this study are at the same dose of $2 \mathrm{~Gy}$, and therefore, $D$ is omitted throughout the text.

- The correlation function in the $\mathrm{Z}$ direction, $C_{z}(i, j)=\left\langle\varepsilon_{i} \varepsilon_{j}\right\rangle$, where $\varepsilon_{i}$ and $\varepsilon_{j}$ are energies deposited in voxels $i$ and $j$, both located on a line parallel to the $\mathrm{Z}$ axis. Owing to the symmetry in the $\mathrm{XY}$ plane within the tally box (parallel beam), the function $C_{z}(i, j)$ does not depend on $\mathrm{X}$ or $\mathrm{Y}$.

- The correlation function in the lateral direction, $C_{x y}(i, j)=\left\langle\varepsilon_{i} \varepsilon_{j}\right\rangle$, where $\varepsilon_{i}$ and $\varepsilon_{j}$ are ener- gies deposited in voxels $i$ and $j$, both of which are located in a plane orthogonal to the $\mathrm{Z}$ axis. Calculations were performed separately for several depths, from 0 to $10 \mu \mathrm{m}$.

- $\quad$ The probability $F_{z}(\varepsilon, i, j)$ of energy deposited in each of the two voxels $i$ and $j$ exceeding $\varepsilon$, with both voxels located on a line parallel to the $\mathrm{Z}$ axis. Like the correlation functions, this quantity characterises spatial correlations of energy depositions. $F_{z}$, however, is more convenient for radiobiological modelling. We did not calculate this function for the lateral direction because our data showed that correlations in that direction were very short-range and therefore negligible. In that case, in the lateral direction, this probability can be found from distributions $f(\varepsilon)$ for individual voxels as a product of probabilities.

The version of Geant4-DNA that is available for download does not include code that calculates the above quantities. For the purposes of this study, we wrote a new user code, the main parts of which were a new source subroutine and a subroutine for tallying the quantities of interest. In Geant4 terminology, these are referred to as the "primary generation action" and "event action", respectively. The physics models that we included were consistent with the "microdosimetry" example included in the standard installation of Geant4. Energy deposition correlation functions were introduced based on the previous work of one of the authors on the application of Monte Carlo techniques to problems of statistical mechanics. ${ }^{32,33}$

\section{Results and Discussion}

\section{Energy deposition patterns at $2 \mathrm{~Gy}$}

Figures 1 and 2 show typical energy deposition patterns at 2 Gy in a $1 \mu \mathrm{m}$ cube for $10 \mathrm{MeV}$ and $100 \mathrm{MeV}$ protons. No neutral hydrogen atoms were produced in these examples. As shown in the figures, energy deposition events were mostly localized very close to proton tracks. Only in relatively rare events were delta electrons produced with energies high enough to travel a distance of more than a few nanometers away from a proton track. The total number of energy deposition events is approximately the same in the two figures. At $10 \mathrm{MeV}$, as expected, the number of energy deposition events per $1 \mu \mathrm{m}$ track segment was much higher than that at 100 $\mathrm{MeV}$. At $10 \mathrm{MeV}$, a notably large fraction of the volume was not affected directly by protons or delta electrons. 


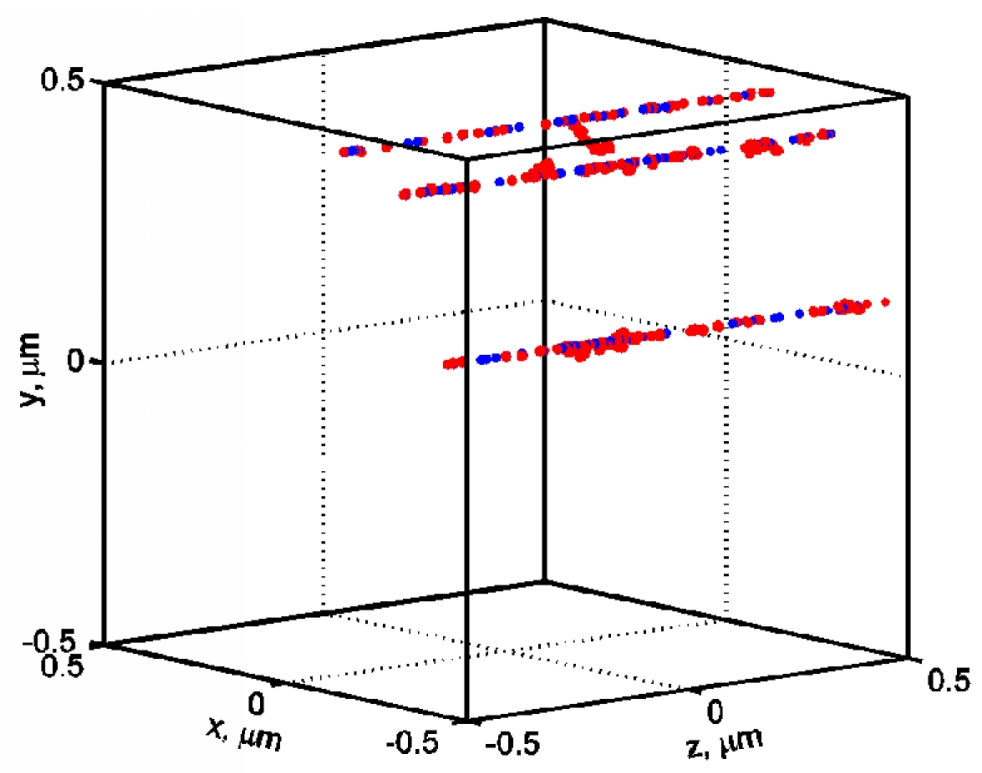

FIG.1: An energy deposition pattern for $10 \mathrm{MeV}$ protons. The number of tracks shown is typical for a dose of $2 \mathrm{~Gy}$. Each dot represents an energy deposition event (e.g., ionization or excitation). Proton tracks are shown in blue, and delta electron tracks are shown in red.

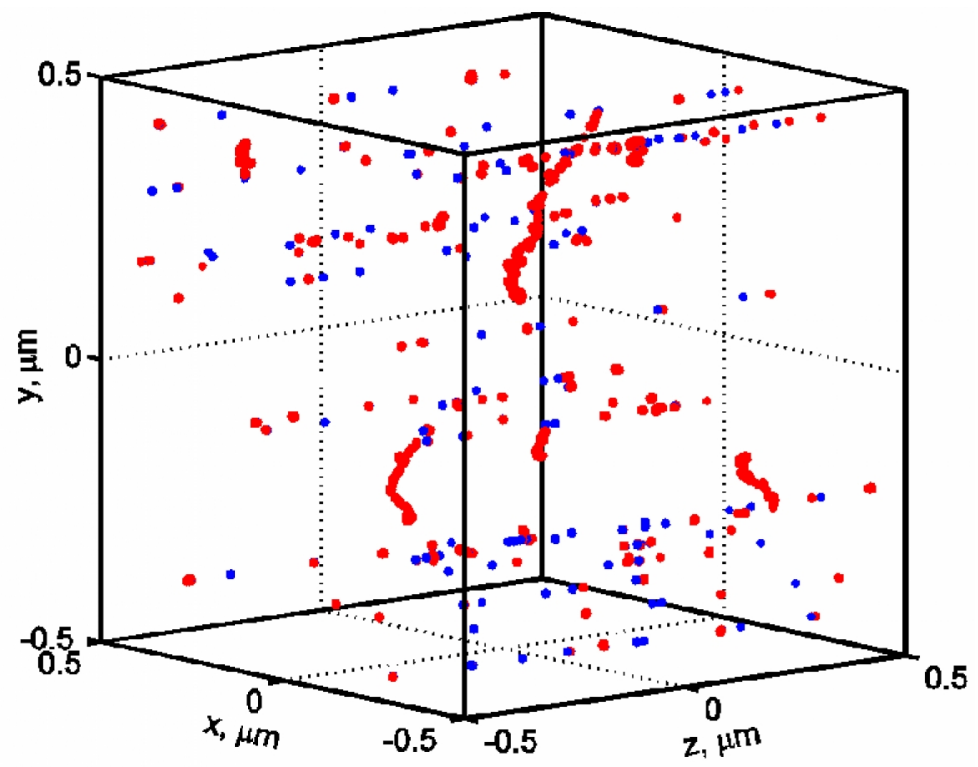

FIG. 2: An energy deposition pattern for $100 \mathrm{MeV}$ protons. The number of tracks shown is typical for a dose of 2 Gy. Each dot represents an energy deposition event (e.g., ionization or excitation). Proton tracks are shown in blue, and delta electron tracks are shown in red.

Carbon tracks of therapeutic energies (not shown) are characterised by an even higher number of energy deposition events per micrometer than $10 \mathrm{MeV}$ protons. Accordingly, the average number of carbon ions needed to deliver a 2 Gy dose is lower. For example, for a carbon beam with an energy of $100 \mathrm{MeV} / \mathrm{u}$, at $2 \mathrm{~Gy}$, the average number of carbon ions entering the tally volume $(1 \mu \mathrm{m} \times 1 \mu \mathrm{m} \times 10 \mu \mathrm{m})$ is 0.48 . This estimate was derived using the total stopping power calcu- lated using SRIM software. ${ }^{34}$ Physical dose per fraction in carbon therapy is usually lower than 2 Gy by a factor of approximately $2-3$ because of the high RBE. The average number of tracks in a volume of this size is thus lower by the same factor. 


\section{Probability distribution of energy deposited in $10 \mathrm{~nm}$ voxels}

All voxels in the tally volume had approximately the same probability distribution of deposited energy $f(\varepsilon)$ because the incident beam was parallel and its properties did not change significantly as it propagated the $10 \mu \mathrm{m}$ distance. Figure 3 shows a histogram estimate of $f(\varepsilon)$ for $10 \mathrm{MeV}$ and $100 \mathrm{MeV}$ protons, as well as $100 \mathrm{MeV} / \mathrm{u}$ carbon ions. The width of each bar in the figure is $1 \mathrm{eV}$. In this figure, the distribution is normalized as follows:

$$
\int_{\varepsilon>0}^{\infty} f(\varepsilon) \mathrm{d} \varepsilon=1 .
$$

This means that $f(\varepsilon)$ is a conditional distribution given the deposited energy is greater than zero.

The first bar represents energy depositions of less than $1 \mathrm{eV}$, excluding zero. These result from excitations of vibrational degrees of freedom of a water molecule by delta electrons. The distinct peaks seen in the figure correspond to discrete levels of electronic excitations. As is evident in the figure, 10 $\mathrm{MeV}$ protons had a higher probability of depositing energy greater than $34 \mathrm{eV}$ than did $100 \mathrm{MeV}$ protons, and carbon ions were more likely to deposit energy greater than $56 \mathrm{eV}$ than were protons. It should be mentioned that this is a conditional probability given $\varepsilon>0$. The mean deposited energies were $47.6 \mathrm{eV}, 38.9 \mathrm{eV}$, and $37.2 \mathrm{eV}$ for $10 \mathrm{MeV}, 50$ $\mathrm{MeV}$, and $100 \mathrm{MeV}$ protons, respectively. The corresponding standard deviations were $62.1 \mathrm{eV}, 56.0 \mathrm{eV}$, and $54.9 \mathrm{eV}$. These results are usually expressed in terms of the frequency mean lineal energy $y_{F}$, and dose mean lineal energy $y_{D}$. It should be noted, however, that $y_{F}$ and $y_{D}$ are normally used to characterise a single event distribution, when a track of only one particle interacts with the SV. Our distributions are multiple-event, that is, the number of particles interacting with a voxel is random and can be greater than 1. Fortunately, for the $10 \mathrm{~nm}$ voxels, at $2 \mathrm{~Gy}$, the most likely number of particles is 0 or 1 . More specifically, the probability of an individual $10 \mathrm{~nm}$ voxel receiving a nonzero energy was $2.57 \times 10^{-4}, 3.10 \times 10^{-4}$, and $3.27 \times 10^{-4}$ for protons of energies 10 $\mathrm{MeV}, 50 \mathrm{MeV}$, and $100 \mathrm{MeV}$, respectively. This leaves a large number of voxels not affected directly by radiation, and, on the other hand, very few voxels will be hit more than once. The calculated $y_{F}$ were $7.15 \mathrm{eV} / \mathrm{nm}, 5.83 \mathrm{eV} / \mathrm{nm}, 5.58$ $\mathrm{eV} / \mathrm{nm}$, and $y_{D}$ were $19.3 \mathrm{eV} / \mathrm{nm}, 17.9 \mathrm{eV} / \mathrm{nm}$, and 17.7 $\mathrm{eV} / \mathrm{nm}$ for $10 \mathrm{MeV}, 50 \mathrm{MeV}$, and $100 \mathrm{MeV}$ protons, respectively. Chmelevsky and Kellerer ${ }^{35}$ reported similar results and also used Monte Carlo simulations. For $5 \mathrm{MeV}$ and 20 $\mathrm{MeV}$ protons, they reported $y_{F}$ of approximately $10.8 \mathrm{eV} / \mathrm{nm}$ and $8.62 \mathrm{eV} / \mathrm{nm}$ (read from a graph), and $y_{D}$ of $20.3 \mathrm{eV} / \mathrm{nm}$ and $17.4 \mathrm{eV} / \mathrm{nm}$. The SV was a sphere of $10 \mathrm{~nm}$ diameter. For the same $\mathrm{SV}$ and $20 \mathrm{MeV}$ protons, a figure in the ICRU Report $36^{27}$ shows $y_{D}$ of approximately $17.8 \mathrm{eV} / \mathrm{nm}$. Our $y_{D}$ agree with the previous studies, whereas our $y_{F}$ is somewhat lower. The latter is known to be more sensitive to the radiation transport model. In Chmelevsky and Kellerer ${ }^{35}$ only ionizations were accounted for. This resulted in higher energy per event because ionizations are associated with higher energy deposits than excitations, either electronic or vibrational.

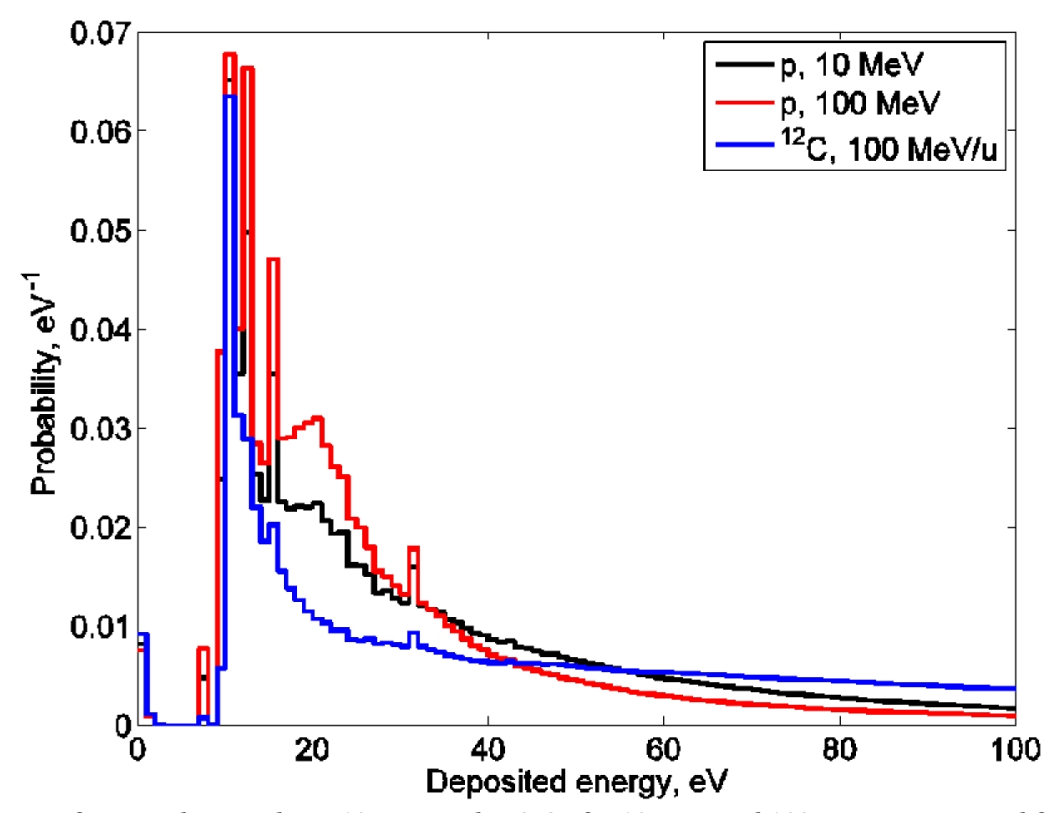

FIG. 3: Probability distribution of energy deposited in a $10 \mathrm{~nm}$ voxel at $2 \mathrm{~Gy}$ for $10 \mathrm{MeV}$ and $100 \mathrm{MeV}$ protons, and for $100 \mathrm{MeV} / \mathrm{u}$ carbon ions. 
Specific energy-volume histogram (zVH)

In radiotherapy, the dose-volume histogram (DVH) is routinely used to describe 3D dose distributions. Here we applied the same idea to microscopic distributions of radiation energy imparted to tissues. We introduce the specific energy-volume histogram, zVH. The specific energy $Z$ is defined as the quotient of energy $\varepsilon$ deposited in an SV by the mass $m$ of tissue in it. ${ }^{27}$ Specific energy has the same units as the absorbed dose (Gy). We define $\mathrm{zVH}$ as the average total volume $\mathrm{V}$ occupied by voxels receiving a specific energy greater than $Z$. The $\mathrm{zVH}$ is a function of $z$ and depends on the dose and voxel size.

The latter means that introducing the $\mathrm{zVH}$ does not completely solve the problem of choosing an appropriate SV size. However, the $\mathrm{zVH}$ somewhat mitigates this problem because the voxel has a different meaning than the SV. The voxel defines the resolution of spatial discretization. Furthermore, $10 \mathrm{~nm}$ is likely an optimal size for a voxel. Choosing a larger size would result in a lower spatial resolution and loss of information about interactions on a nanoscale. Voxels smaller than $10 \mathrm{~nm}$ would certainly provide a more detailed description of the physical stage of radiation effects on tissues. However, the physical stage is followed by the chemical stage, which is associated with delocalization of deposited energy through the diffusion of water radiolysis products that travel distances exceeding a few nanometers. ${ }^{36}$ Considering the computational cost and large uncertainties in the cross-sectional data at low energies near the end of electron track, the benefits of a further reduction of the voxel size are questionable.

Calculated zVH for $10 \mathrm{MeV}$ and $100 \mathrm{MeV}$ protons, and 100 $\mathrm{MeV} / \mathrm{u}$ carbon beams are shown in Figure 4. Compared with the $\mathrm{DVH}$, the shape of the $\mathrm{zVH}$ resembled DVH for organs at risk. However, the scales of both axes differed considerably. Although the dose shown in Figure 4 was only 2 Gy, in terms of the specific energy, all three curves extended beyond 40 $\mathrm{kGy}$. At the same time, the figure shows that only a very small fraction of tissue volume received any energy directly from either proton or carbon beams. Qualitatively, this result is consistent with the energy deposition patterns shown in Figures 1 and 2. Finally, at exactly the same dose for the all three beams, the three $\mathrm{zVH}$ curves were distinctly different. Given that the biological effects would also be different, this suggests that models can be developed that correlate properties of the $\mathrm{zVH}$ with tissue response. For example, similar to the standard approach used in microdosimetry ${ }^{24}$, the biological effect $E$ at a given dose $D$ can be represented as a linear functional of the $\mathrm{zVH}$ :

$$
E(D)=\int_{0}^{\infty} z V H(z \mid D) w(z) \mathrm{d} z,
$$

where function $w(z)$ describes the response of a biological system and can be determined by fitting Eq. (2) to a set of experimental data for different radiation fields. Alternatively, or in addition to Eq. (2), simple metrics associated with the $\mathrm{zVH}$ can be tested for correlation with tissue response. Such metrics based on the DVH are used routinely in radiotherapy for evaluating treatment plans and predicting treatment outcomes. By analogy to the metrics used in radiotherapy, metrics based on the $\mathrm{zVH}$ may include, for example, $\mathrm{V}$ (20 $\mathrm{kGy}$ ), that is, the volume receiving $z>20 \mathrm{kGy}$. Compared with the dose dependent multiple-event microdosimetric spectrum $f(z \mid D)$, the zVH presents data in a format more familiar to radiotherapy professionals and clearly shows the volume not affected directly by radiation ( $z=0$ ). The RBE can be found from Eq. (2) simply as the ratio of doses that produce the same effect, that is $R B E=D_{1} / D_{2}$, where $D_{1}$ and $D_{2}$ satisfy equation $E\left(D_{1}\right)=E\left(D_{2}\right)$.

\section{Correlation functions}

Figure 5 shows correlation functions $C_{z}$ for energy deposited in two voxels located on a line parallel to the $\mathrm{Z}$ axis. The data shown are for $10 \mathrm{MeV}$ and $100 \mathrm{MeV}$ proton beams, and a 100 $\mathrm{MeV} / \mathrm{u}$ carbon beam. As illustrated in the figure, all three curves were very flat, which indicates that correlations did not attenuate over distances exceeding $10 \mu \mathrm{m}$. Clearly, correlations remain strong at distances on the order of the distance between nuclei of two neighbouring cells, and likely at even larger distances, which results in an increased probability of a proton or especially a carbon ion producing a cluster of damaged cells along its track. This effect may alter how the affected cells respond to radiation and, therefore, should be considered when modelling tissue response. The spatial resolution of the data was $10 \mathrm{~nm}$. Small fluctuations in the data were due to the statistical uncertainties of Monte Carlo simulations. The spike at the graph origin represents self-correlation, $\left\langle\varepsilon_{i} \varepsilon_{j}\right\rangle$, where $i=j$. All three self-correlation values shown in Figure 5 are consistent with the standard deviations of deposited energies reported earlier.

In contrast, correlations in the lateral direction attenuate very rapidly. This can be seen in Figure 6, which shows the correlation function $C_{x y}$. To estimate the correlation radius, $R_{x y}, C_{x y}$ as a function of distance $r$ between two voxels was approximated through a least squares fit by the exponent $\exp \left(-r / R_{x y}\right)$. The best-fit values of $R_{x y}$ for $10 \mathrm{MeV}, 50$ $\mathrm{MeV}$, and $100 \mathrm{MeV}$ protons were $12.3 \mathrm{~nm}, 11.4 \mathrm{~nm}$, and 10.8 $\mathrm{nm}$, respectively. For $100 \mathrm{MeV} / \mathrm{u}$ carbon ions, the $R_{x y}$ estimate was $12.8 \mathrm{~nm}$. Apparently, the correlation radius tends to increase with increasing LET. The fit was limited to a distance of less than $100 \mathrm{~nm}$. Beyond $100 \mathrm{~nm}$, the correlation function approached its asymptotic value, indicated in the figure by a horizontal dashed line. This value represents a complete 
attenuation of correlations, that is $\left\langle\varepsilon_{i} \varepsilon_{j}\right\rangle=\left\langle\varepsilon_{i}\right\rangle\left\langle\varepsilon_{j}\right\rangle$. The asymptotic value was the same for all three beams shown because $\left\langle\varepsilon_{i}\right\rangle$ is proportional to the dose, which was the same (2 Gy) for all three beams. The asymptotic value $\left\langle\varepsilon_{i}\right\rangle\left\langle\varepsilon_{j}\right\rangle$ for the dose of 2 Gy was calculated completely independently from the Monte Carlo simulations. Finally, the statistical uncertainties were rather large at large distances because of a small probability of having non-zero deposited energies in both voxels.

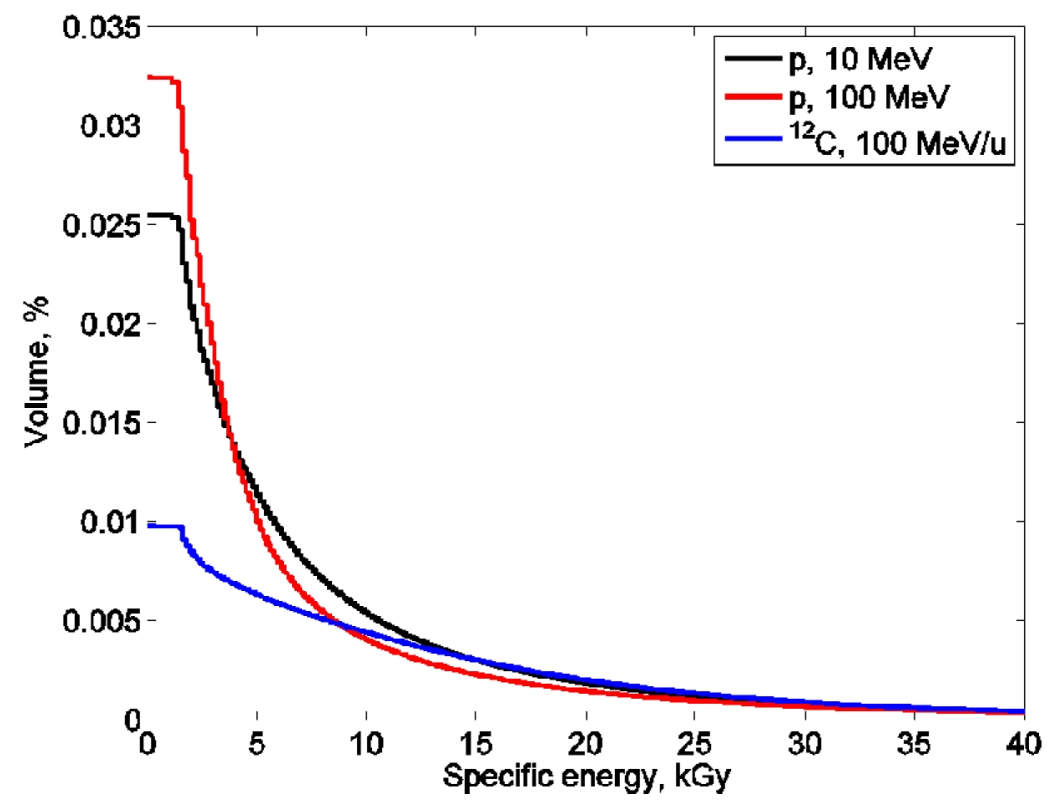

FIG. 4: The specific energy-volume histogram, zVH, for $10 \mathrm{MeV}$ and $100 \mathrm{MeV}$ protons and for $100 \mathrm{MeV} / \mathrm{u}$ carbon ions.

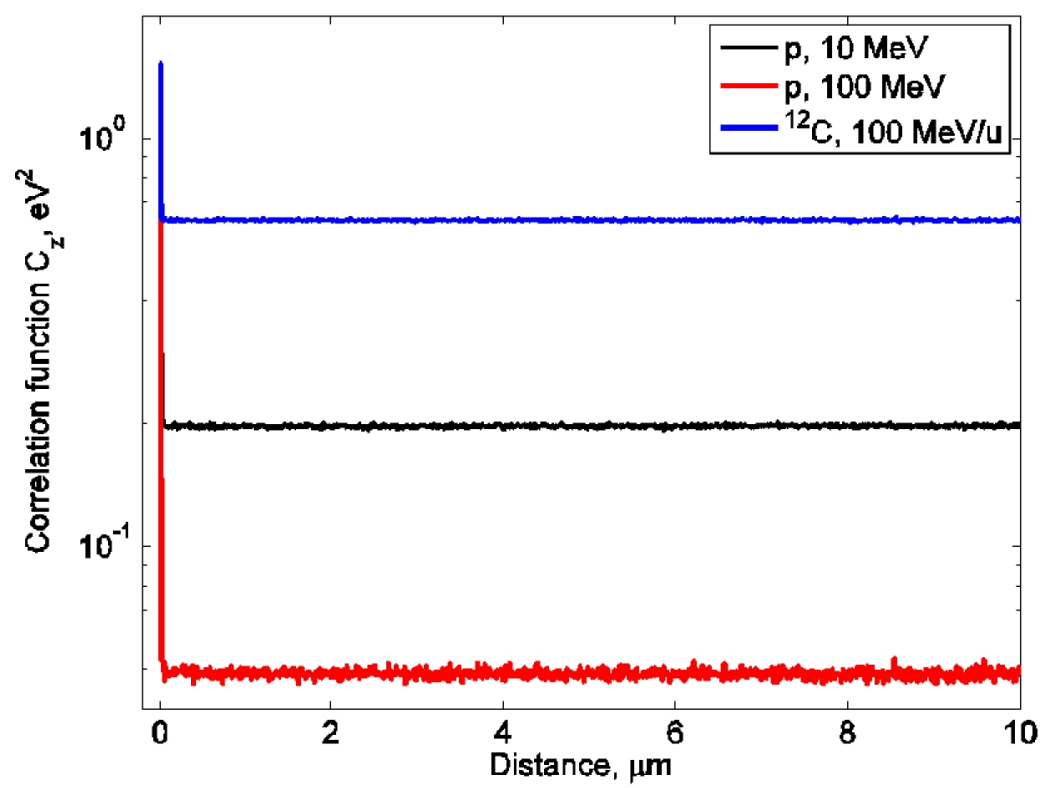

FIG.5: Correlation functions in the direction parallel to particle tracks ( $\mathrm{Z}$ axis), $C_{z}$, for $10 \mathrm{MeV}$ and $100 \mathrm{MeV}$ protons and for $100 \mathrm{MeV} / \mathrm{u}$ carbon ions. 


\section{Probability distribution $\mathbf{F}_{\mathbf{z}}(\boldsymbol{\varepsilon}, \mathbf{i}, \mathbf{j})$}

Similarly to the correlation function $C_{z}$, the probability distribution $F_{z}(\varepsilon, i, j)$ characterises correlations of energy deposition events along the direction of a particle track. Given that correlations in the lateral direction are virtually nonexistent, such characterisation is adequate for modelling tissue response. The advantage of $F_{z}(\varepsilon, i, j)$ is that it explicitly specifies the probabilities of two voxels receiving a certain amount of energy. This information can be correlated with tissue response through a model (for example, phenomenological) similar to the one given by Eq. (2). Alternatively, the more conventional joint probability distribution $F_{z}\left(\varepsilon_{1}, \varepsilon_{2}, i, j\right)$ could be used for that purpose. However, it is less convenient because it has an extra argument, and we do not anticipate that the extra information this argument provides will profoundly improve the model.

Figure 7 shows the distribution $F_{z}(\varepsilon, i, j)$, with solid lines for two voxels separated by a distance of $1 \mu \mathrm{m}$, for $10 \mathrm{MeV}$ and $100 \mathrm{MeV}$ proton beams and for a $100 \mathrm{MeV} / \mathrm{u}$ carbon ion beam. We found that the distribution did not depend on the distance between two voxels of up to a distance of $10 \mu \mathrm{m}$, which was the maximum distance that we tested. This is consistent with the behaviour of the correlation functions discussed earlier. As shown in the figure, the probability of each of the two voxels receiving high energy increased with increasing LET. Furthermore, to demonstrate the profound effect correlations have on this distribution, we also calculated it assuming that the two voxels are completely independent and using the distribution $f(\varepsilon)$ for an individual voxel. This result is shown in Figure 7 with dashed lines. These calculated probabilities were orders of magnitude lower. Therefore, to simplify comparisons, we show these probabilities multiplied by a factor of $10^{2}, 10^{3}$, and $10^{4}$ for 100 $\mathrm{MeV}$ protons, $10 \mathrm{MeV}$ protons, and $100 \mathrm{MeV} / \mathrm{u}$ carbon ions, respectively. Clearly, assuming statistical independence among voxels may result in significant errors, even when voxels are separated by a large, on the cellular scale, distance of $10 \mu \mathrm{m}$.

A comparison of Figure 7 and Figure 4 shows that the order of the three curves near the origin was different. This is because the data shown in Figure 7 were a result of the interplay of two factors. The first factor is the probability of a nonzero energy deposit in the first of the two voxels, the maximum of which occurs for $100 \mathrm{MeV}$ protons, as is shown in Figure 4. The second factor is the conditional probability of a nonzero energy deposit in the second voxel, given that a nonzero energy was deposited in the first voxel. This conditional probability is maximal for carbon beams because they show a stronger correlation (Figure 5).

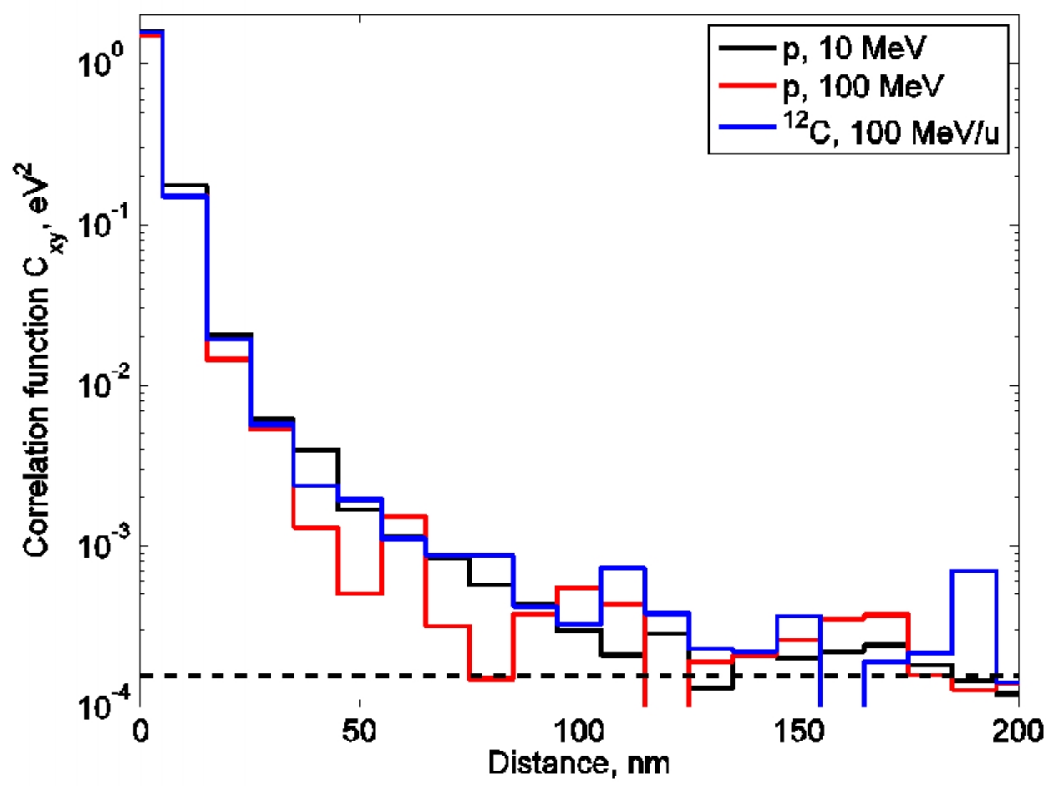

FIG. 6: Correlation functions in the lateral direction with respect to particle tracks (XY plane), $C_{x y}$, for $10 \mathrm{MeV}$ and $100 \mathrm{MeV}$ protons and for $100 \mathrm{MeV} / \mathrm{u}$ carbon ions. The horizontal dashed line indicates the asymptotic value, which represents a complete attenuation of correlations at large distances. 


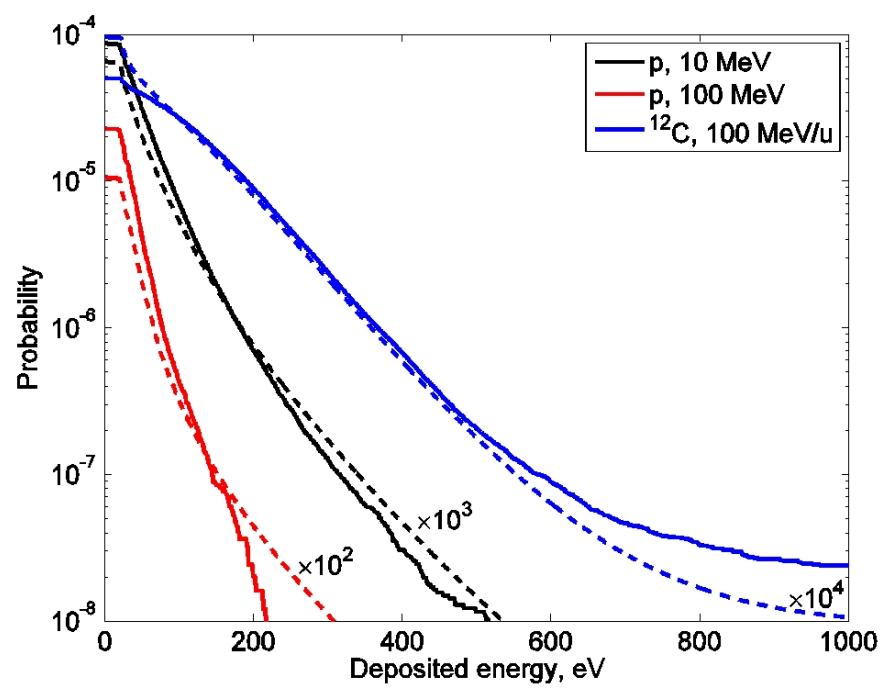

FIG. 7: The probability $F_{z}(\varepsilon, i, j)$ of energy deposited in each of the two voxels $i$ and $j$ exceeding $\varepsilon$ (solid lines). Both voxels are located on the $\mathrm{Z}$ axis and separated by a distance of $1 \mu \mathrm{m}$. Data are shown for $10 \mathrm{MeV}$ and $100 \mathrm{MeV}$ protons and $100 \mathrm{MeV} / \mathrm{u}$ carbon ions. The dashed lines show the same probability calculated assuming statistical independence. This assumption results in underestimation by orders of magnitude. Thus, to simplify comparison, these results are shown multiplied by a factor of $10^{2}, 10^{3}$, and $10^{4}$, as indicated in the figure.

\section{Conclusions}

On the spatial scale of subcellular structures, radiation fields are rather complex and should be described in statistical terms. Microdosimetry has a formalism developed for that purpose. However, the amount of detail needed to predict tissue response in radiotherapy remains unclear. In this study, we extended the microdosimetric approach somewhat to include characteristics that may be important for that application and to present them in a more conventional radiotherapy format. We have shown that in some realistic scenarios, both the spatial correlations of deposited energy and the volume not directly affected by radiation can be significant. We hypothesize that these variables may be important when modelling normal tissue complications or tumor control. This may be particularly important considering the growing evidence indicating that cells in tissues do not respond to radiation independent of one another. If, for example, spatial correlations in radiation fields indeed result in the formation of clusters of damaged cells, the tissue may respond differently than it would if damaged cells were spread more uniformly throughout the tissue. All examples presented in this paper concern hadron therapy because the effects described herein are more prominent, and the radiobiology of tissue response more directly affects treatment optimization and, for this reason, remains a very active area of research.

\section{Conflict of interest}

The authors declare that they have no conflicts of interest. The authors alone are responsible for the content and writing of the paper.

\section{References}

1. Paganetti H, Schmitz T. The influence of the beam modulation technique on dose and RBE in proton radiation therapy. Phys Med Biol 1996; 41:1649-63.

2. Coutrakon G, Cortese J, Ghebremedhin A, et al. Microdosimetry spectra of the Loma Linda proton beam and relative biological effectiveness comparisons. Med Phys 1997; 24:1499-506.

3. Paganetti H, Olko $\mathrm{P}$, Kobus $\mathrm{H}$, et al. Calculation of relative biological effectiveness for proton beams using biological weighting functions. Int J Radiat Oncol Biol Phys 1997; 37:719-29.

4. Endo S, Tanaka K, Ishikawa M, et al. Microdosimetric evaluation of the $400 \mathrm{MeV} /$ nucleon carbon beam at HIMAC. Med Phys 2005; 32:3843-8.

5. Nose H, Kase Y, Matsufuji N, Kanai T. Field size effect of radiation quality in carbon therapy using passive method. Med Phys 2009; 36:870-5.

6. Inaniwa T, Furukawa T, Kase Y, et al. Treatment planning for a scanned carbon beam with a modified microdosimetric kinetic model. Phys Med Biol 2010; 55:6721-37.

7. Martino G, Durante M, Schardt D. Microdosimetry measurements characterizing the radiation fields of 300 $\mathrm{MeV} / \mathrm{u} 12 \mathrm{C}$ and $185 \mathrm{MeV} / \mathrm{u} 7 \mathrm{Li}$ pencil beams stopping in water. Phys Med Biol 2010; 55:3441-9.

8. Tsuda S, Sato T, Takahashi F, et al. Measurement of microdosimetric spectra with a wall-less tissue-equivalent proportional counter for a $290 \mathrm{MeV} / \mathrm{u}$ 12C beam. Phys Med Biol 2010; 55:5089-101.

9. Endo S, Tanaka K, Takada M, et al. Microdosimetric study for secondary neutrons in phantom produced by a 
290 MeV/nucleon carbon beam. Med Phys 2007; 34:3571-8.

10. Wroe A, Rosenfeld A, Schulte R. Out-of-field dose equivalents delivered by proton therapy of prostate cancer. Med Phys 2007; 34:3449-56.

11. Yonai S, Kase Y, Matsufuji N, et al. Measurement of absorbed dose, quality factor, and dose equivalent in water phantom outside of the irradiation field in passive carbon-ion and proton radiotherapies. Med Phys 2010; 37:4046-55.

12. Wuu CS, Kliauga $\mathrm{P}$, Zaider $\mathrm{M}$, Amols HI. Microdosimetric evaluation of relative biological effectiveness for 103Pd, 125I, 241Am, and 192Ir brachytherapy sources. Int I Radiat Oncol Biol Phys 1996; 36:689-97.

13. Taschereau R, Rene R, Pouliot J. Relative biological effectiveness enhancement of a 125I, brachytherapy seed with characteristic $\mathrm{x}$ rays from its constitutive materials. Med Phys 2002; 29:1397-02.

14. Reniers B, Vynckier S, Verhaegen F. Theoretical analysis of microdosimetric spectra and cluster formation for 103Pd and 125I photon emitters. Phys Med Biol 2004; 49:3781-95.

15. Zellmer DL, Almos HI. Microdosimetric single-event spectra for megavoltage electrons. Med Phys 1990; 17:596-01.

16. Okamoto H, Kohno T, Kanai T, et al. Microdosimetric study on influence of low energy photons on relative biological effectiveness under therapeutic conditions using 6 MV linac. Med Phys 2011; 38:4714-22.

17. Hawkins RB. A Microdosimetric-Kinetic model for the effect of non-Poisson distribution of lethal lesions on the variation of RBE with LET. Radiat Res 2003; 160:61-9.

18. Kase Y, Kanai T, Matsumoto Y, et al. Microdosimetric measurements and estimation of human cell survival for heavy-ion beams. Radiat Res 2006; 166:629-38.

19. Kase Y, Kanai T, Matsufuji N, et al. Biophysical calculation of cell survival probabilities using amorphous track structure models for heavy-ion irradiation. Phys Med Biol 2008; 53:37-59.

20. Hanin LG, Zaider M. Cell-survival probability at large doses: an alternative to the linear-quadratic model. Phys Med Biol 2010; 55:4687-702.

21. Vassiliev ON. Formulation of the multi-hit model with a non-Poisson distribution of hits. Int J Radiat Oncol Biol Phys 2012; 83:1311-16.

22. Lindborg L, Hultqvist M, Tedgren AC, Nikjoo H. Lineal energy and radiation quality in radiation therapy: model calculations and comparison with experiment. Phys Med Biol 2013; 58:3089-105.

23. Li XA, Alber M, Deasy JO, Jackson A, et al. The use and QA of biologically related models for treatment planning: Short report of the TG-166 of the therapy physics committee of the AAPM. Med Phys 2012; 39:1386-409.

24. Brenner DJ, Zaider M. Estimating RBEs at clinical doses from microdosimetric spectra. Med Phys 1998; 25:1055-57.

25. Pihet $\mathrm{P}$, Menzel HG. Response to the Letter to the Editor "Estimating RBEs at clinical doses from microdosimetric spectra" [Med. Phys. 25, 1055 (1998)]. Med Phys 1999; 26:848-52.

26. Blyth BJ, Sykes PJ. Radiation-induced bystander effects: what are they, and how relevant are they to human radiation exposures? Radiat Res 2011; 176:139-57.

27. ICRU 1983 Microdosimetry ICRU Report 36 (Bethesda, MD: International Commission on Radiation Units and Measurements)

28. Villagrasa C, Francis Z, Incerti S. Physical models implemented in the Geant4-DNA extension of the Geant4 toolkit for calculating initial radiation damage at the molecular level. Radiat Protect Dosim 2011; 143:214-18.

29. Dingfelder $M$, Inokuti $M$, Paretzke HG. Inelastic-collision cross sections of liquid water for interactions of energetic protons. Radiat Phys Chem 2000; 59:255-75.

30. Francis Z, Incerti S, Karamitros M, et al. Stopping power and ranges of electrons, protons and alpha particles in liquid water using the Geant4-DNA package. Nucl Instr Meth Phys Res B 2011; 269:2307-11.

31. Nikjoo H, Lindborg L. RBE of low energy electrons and photons. Phys Med Biol 2010; 55:R65-109.

32. Vassiliev ON, Rojdestvenski IV, Cottam MG. Quantum Monte-Carlo study of the Heisenberg chain with long-range ferromagnetic interactions. Physica A 2001; 294:139-54.

33. Vassiliev ON, Matsen MW. Fluctuation effects in block copolymer melts. J Chem Phys 2003; 118:7700-13.

34. Ziegler JF, Ziegler MD, Biersack JP. SRIM-the stopping and range of ions in matter (2010). Nucl Instrum Methods Phys Res B 2010; 268:1818-23.

35. Chmelevsky D, Kellerer AM. Computation of microdosimetric distributions for small sites. Radiat Environ Biophys 1977; 14:123-36.

36. Friedland W, Jacob P, Paretzke HG, Merzagora M, Ottolenghi A. Simulation of DNA fragment distributions after irradiation with photons. Radiat Environ Biophys 1999; 38:39-47. 